Communications in Physics, Vol. 20, No. 2 (2010), pp. 181-192

\title{
EXTENSIVE AIR SHOWERS AND THE LPM EFFECT
}

\author{
LION ALIO, PHAM NGOC DIEP, PHAM NGOC DONG, DO THI HOAI, \\ PHAM THI TUYET NHUNG, PIERRE DARRIULAT, AND NGUYEN THI THAO \\ VATLY, Institute for Nuclear Science and Technology
}

\begin{abstract}
A very simple model of the longitudinal development of electromagnetic showers is presented, allowing for easy calculations at the highest energies where usual simulation codes become impracticable because of the enormous multiplicity of secondary shower particles. Shower profiles are described in terms of a few parameters having Gaussian fluctuations. The model is applied to the impact of the Landau-Pomeranchuk-Migdal effect on the shower development, which is found negligible in practical cases.
\end{abstract}

\section{INTRODUCTION}

Cosmic rays are ionized nuclei that have been accelerated in space up to high energies and reach the Earth. When entering the atmosphere they interact with it and produce cascades of new particles called extensive air showers. Cosmic rays may reach up to around $10^{20} \mathrm{eV}$, an energy above which they interact with the cold $(2.7 \mathrm{~K})$ photons of the cosmic microwave background to produce pions, thereby losing energy in the process [1]. At the highest energies a quantum effect, referred to as the Landau-Pomeranchuk-Migdal (LPM) effect [2], causes a reduction of the cross-sections associated with elementary processes at play in the development of electron or photon induced showers, essentially bremsstrahlung and pair creation. As a result, the shower develops over a longer distance than would be predicted by a simulation ignoring the LPM effect. This is of particular importance for the identification of the nature of the primary cosmic rays as the main difference between showers induced by a proton and by an iron nucleus of same energy is that, on average, the latter starts developing at higher altitudes than the former.

The aim of the present work is to evaluate the effect of the LPM reduction on the development of extensive air showers. As it acts exclusively on showers induced by electrons or by photons it affects essentially those induced by the two-photon decay of neutral pions produced in the first hadronic interaction in the upper atmosphere.

The article is organized as follows: Section 2 describes the development of electromagnetic showers and the method used for its simulation; Section 3 gives a brief account of the physics of the LPM effect and the evidence for it and Section 4 presents the results. 


\section{LONGITUDINAL SHOWER DEVELOPMENT}

\section{II.1. The Method}

The present work uses a simple model of the longitudinal development of electron and photon showers, retaining only pair creation and bremsstrahlung as relevant elementary processes. At very high energies, showers contain so many particles that it is impracticable to follow each of them in a simulation. Most existing codes deal with this problem by using statistical approximations (sampling, averaging, thinning, etc.). The approach used here is different: as soon as a shower particle, electron or photon, has energy lower than some threshold, it is replaced by a parameterized subshower profile, considerably reducing the complexity of the problem. All what needs to be done is then to devise a proper parameterization of the shower profile and to calculate the dependence on energy of the parameters. In practice, the mean and $r m s$ values of the parameters are calculated once for all as a function of energy and the subshowers are generated accordingly with random Gaussian fluctuations of the parameters having the proper means and variances.

\section{II.2. Elementary Processes}

Showers may be initiated by an electron (or positron, here electron is to be understood as electron or positron) or a photon and any other particle that may be created in the cascade (such as $\mu^{+} \mu^{-}$pairs from photon conversion) is ignored. Moreover the only processes considered are pair creation in the case of photons and bremsstrahlung in the case of electrons, implying that Compton scattering, photoelectric effect, and other processes that are important at lower energies are not taken into account.

To a very good approximation, the probability $d^{2} P$ for a photon of energy $E$ to convert in a medium of radiation length $X_{0}$ over a thickness $d x=X_{0} d t$ ( $t$ has no dimension, $d x$ and $X_{0}$ are measured in $\mathrm{g} / \mathrm{cm}^{2}$ ), into a pair having an electron of energy in the interval $[\eta, \eta+d \eta]$ (the positron energy being in the interval $[E-\eta, E-\eta-d \eta]$ ) is (writing $u=\eta / E$ )

$$
d^{2} P=\{1-4 u(1-u) / 3\} d u d t
$$

The radiation length in air is $36 \mathrm{~g} / \mathrm{cm}^{2}$.

The dependence of $E d^{2} P / d \eta d t$ on $u$ is displayed in Figure 1 (right). It has a parabolic shape with a minimum of $2 / 3$ corresponding to the symmetric case (electron and positron having equal energies). It is symmetric in the exchange of the electron and positron ( $u$ becoming $1-u$ ). Integration over $u$ gives $d P / d t=7 / 9$ : the photon distribution over the thickness traversed, $x=t X_{0}$, is an exponential of the form $\exp (-7 / 9 t)$.

In the case of an incident electron of energy $E$, the probability $d^{2} P$ to radiate, over a distance $d x=X_{0} d t$, a photon having an energy in the interval $[\eta, \eta+d \eta]$ is, to a good approximation,

$$
d^{2} P=\left\{4(1-u) / 3+u^{2}\right\} d t d \eta / \eta
$$

It is illustrated in Figure 1 (left) where $d^{2} P /(d t d \eta / \eta)$ is shown against $u=\eta / E$. It reaches a minimum at $8 / 9$ for $u=2 / 3$ while being unity when $u=1$ and being $4 / 3$ when $u=0$. The total energy bremsstrahled per interval $d t$ is

$$
\int \eta d^{2} P=\{4 E / 3-4 E / 3 / 2+E / 3\} d t=E d t .
$$



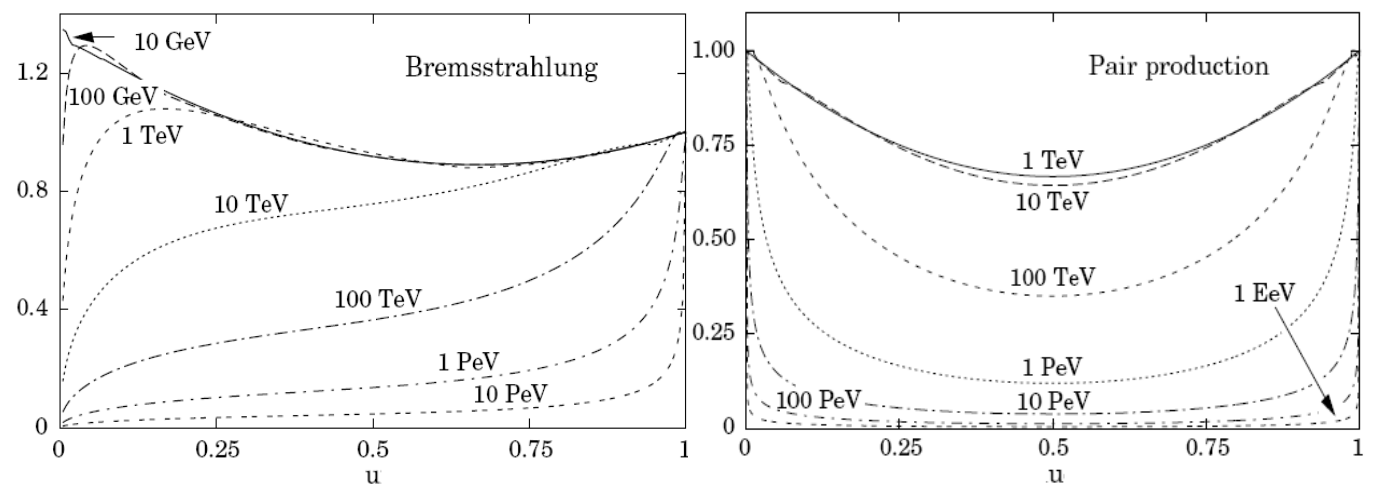

Fig. 1. Left: Differential bremsstrahlung probability per unit of radiation length and per $d \eta / \eta$ as a function of the fractional energy taken away by the photon. Right: Differential pair creation probability per unit of radiation length as a function of the fractional energy taken by the electron. Full lines are without and dashed lines with LPM reduction (from $100 \mathrm{GeV}$ to $10 \mathrm{PeV}$ for bremsstrahlung and from $10 \mathrm{TeV}$ to $1 \mathrm{EeV}$ for pair creation in steps of a factor 10).

The remaining energy has therefore an exponential dependence over the thickness $x=t X_{0}$ traversed of the form $e^{-t}$. However, the number of photons bremsstrahled is infinite, an infinite number of zero energy photons being radiated. Introducing a cut-off $\epsilon$, the number of radiated photons having energy in excess of $\epsilon$ is obtained by integration over $\eta$ between $\epsilon$ and $E$ :

$$
d N=\left\{(4 / 3) \ln (E / \epsilon)-5 / 6+(4 / 3) \epsilon / E-(\epsilon / E)^{2} / 2\right\} d t
$$

The multiplication of particles in the cascade is counteracted by the energy losses which they suffer. The critical energy, $E_{c}$, is defined as the energy where an electron loses as much energy by ionization as it does by radiation. It is equal to $80 \mathrm{MeV}$ in air. The strategy adopted here is to consider bremsstrahlung explicitly only for electrons having an energy in excess of $E_{c}$, namely setting $\epsilon=E_{c}$ in Relation 4 . At $E=10^{21} \mathrm{eV}$ with $\epsilon=E_{c}=80 \mathrm{MeV}$ and $d x=0.01 X_{0}$, Relation 4 gives $d N \sim\{20-\ln 80-5 / 6\} 0.01 \sim 0.15$. Multiple photon radiation can therefore be safely neglected when using such small steps of 0.01 radiation lengths.

The energy radiated in the form of photons of energy lower than $E_{c}$ is, in such a step:

$$
\left.d E=0.01\left\{4\left(E_{c} / E\right) / 3-2\left(E_{c} / E\right)^{2} / 3+\left(E_{c} / E\right)^{3} / 3\right)\right\} E
$$

The electron energy loss is calculated in each slice $d x=0.01 X_{0}$ as the sum of the latter and of the ionization loss:

$$
d E / d x=0.01 E_{c}\left(1+0.15 \log _{10}\left[E / E_{c}\right]\right) d t
$$

In addition any particle, electron or photon, having energy lower than $1.5 \mathrm{MeV}$ is made to stop and to deposit its energy in the shower. Both this energy and the energy loss 
calculated using Relation 6 are deposited over two radiation lengths with a profile having a maximum at one radiation length.

The model has been checked against the result of a detailed simulation [3] for 30 $\mathrm{GeV}$ electrons in iron $\left(E_{c}=20 \mathrm{MeV}\right)$. The result is displayed in Figure 2 and shows quite good agreement given the high energy approximation used here.

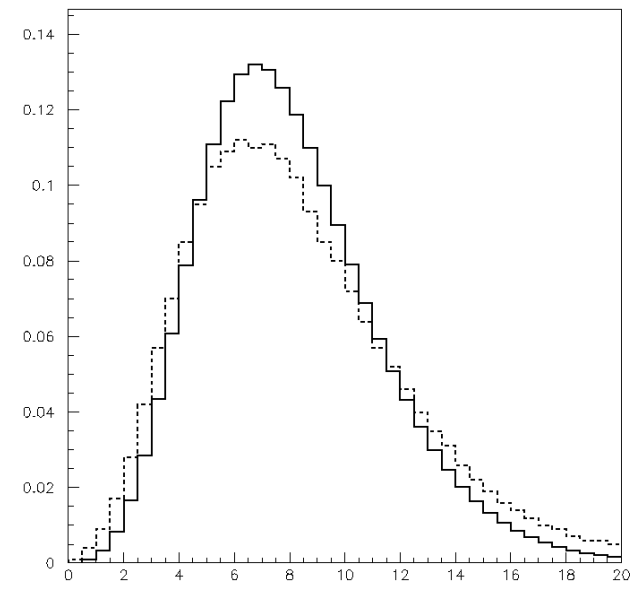

Fig. 2. Average longitudinal profile of a shower induced by a $30 \mathrm{GeV}$ electron in iron: full line, result of the present simulation; dotted line: EGS4 result [3]. Units are radiation lengths.

\section{II.3. Parameterization of The Profile}

The form used here to parameterize the longitudinal shower profile is the standard Gaisser-Hillas function [4]

$$
\ln S=\ln S_{\max }+\left\{\left[X_{\max }-X_{0}\right] / w\right\}\left\{1+\ln \left(\left[X-X^{*}\right] /\left[X_{\max }-X^{*}\right]\right)\right\}
$$

where $S$ is the density of charged particles at depth $X$ in the medium. In practice, $S d X$ may be the sum of the charged particle track lengths in the transverse shower slice between $X$ and $X+d X$, or the energy ionization loss in that same slice, or even the amount of Cherenkov light produced in that same slice. At high energies, all three distributions are expected to have very similar shapes. The depth variable $X$ is measured in $\mathrm{g} / \mathrm{cm}^{2}$ with $d X$ being the product of the local density by the thickness of the slice. In atmospheric air the dependence of density on altitude distorts $X$ with respect to actual distances.

The quantity $X^{*}$ defines where the shower, understood as its charged particle components, starts developing. In the case of a photon, it starts at the location of the first pair creation while in the case of an electron it starts at $X^{*}=0$. Obviously, once started, the shower develops independently from $X^{*}$ and $S$ depends explicitly on $X-X^{*}$. It is therefore sufficient to consider showers induced by electrons, i.e. having $X^{*}=0$.

Taking $S_{\max }$ and $X_{\max }$ as units, one defines reduced variables $\eta=S / S_{\max }$ and $\xi=X / X_{\max }$. The reduced profile then reads $\eta=\{\xi \exp (1-\xi)\}^{\delta}$ and depends on a single parameter $\delta=X_{\max } / w$. Equivalently, $\ln \eta=\delta(\ln \xi+1-\xi)$. 


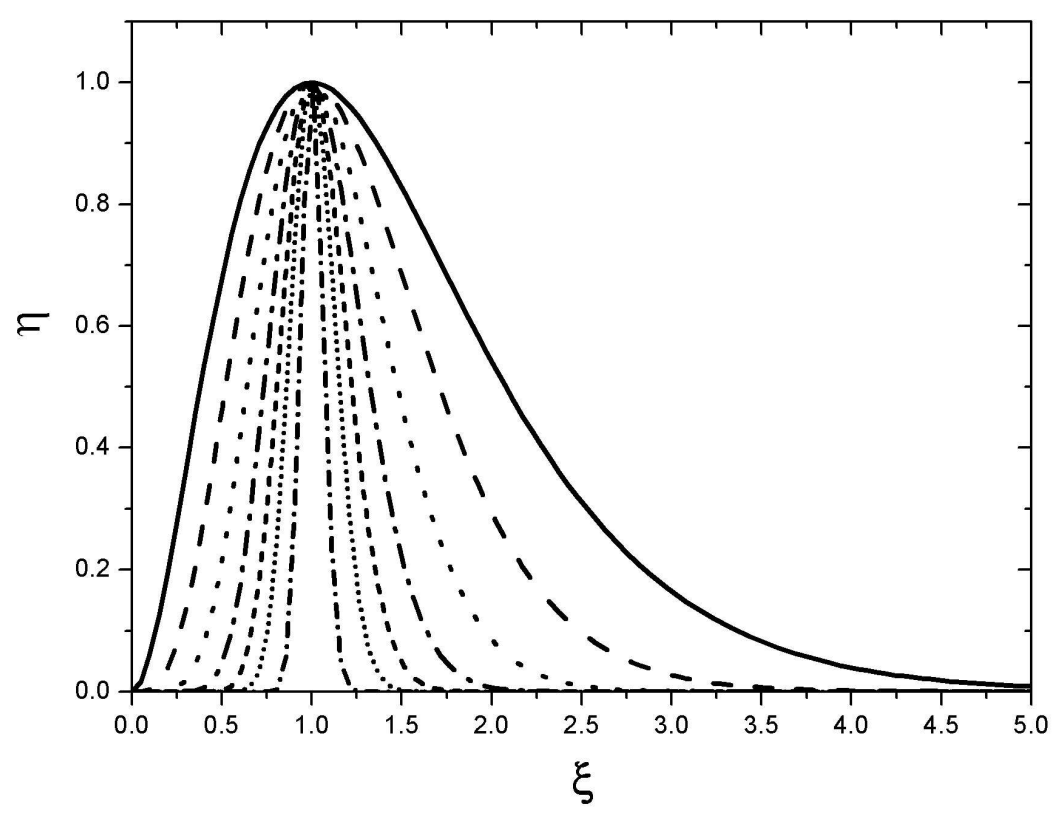

Fig. 3. Reduced profiles for different values of $\delta(2,4,8,16,32,64$ and 256).

The reduced profile starts at 0 at origin as $\xi^{\delta}$ and approaches 0 again when $\xi \rightarrow \infty$. Differentiating gives $d \eta / d \xi=\eta \delta(1 / \xi-1)$ which cancels for $\xi=1$ where $\eta$ reaches its maximum value, 1 , independently from $\delta$. Therefore, the real profile reaches its maximum value $S_{\max }$ at $X=X_{\max }$ which justifies their names. The second derivative, $d^{2} \eta / d^{2} \xi=$ $\eta \delta^{2}(1 / \xi-1)^{2}-\eta \delta / \xi^{2}$ cancels for $\delta(1 / \xi-1)^{2}=1 / \xi^{2}$ or $\xi=1 \pm 1 / \sqrt{\delta}$. While the turning points are equidistant from $\xi=1$ the profile is not at all symmetric around this value. As illustrated in Figure 3 it is significantly skewed, the more the larger $\delta$. As $\delta>1$, the profile starts tangent to the $\xi$ axis. Analytic expressions of the mean, rms and integral values are given in Table 1 below, both for the reduced profile and the real one.

Table 1. Gaisser-Hillas parameters for an electron $\left(X^{*}=0\right)$.

\begin{tabular}{ccc}
\hline \hline Parameter & Reduced profile & Real profile \\
\hline Mean value & $1+1 / \delta$ & $X_{0}+X_{\max }\left(1+w / X_{\max }\right)$ \\
\hline Rms value & $\sqrt{1+\delta} / \delta$ & $\sqrt{\left(w+X_{\max }\right) w}$ \\
\hline Integral & $J(\delta)=e^{\delta} \Gamma(\delta+1) / \delta^{\delta+1}$ & $S_{\max } X_{\max } J\left(X_{\max } / w\right)$ \\
\hline \hline
\end{tabular}

The knowledge of $\langle X\rangle$ and of $\operatorname{Rms}(X)$ fixes $w$ and $X_{\max }$. The knowledge of $\Sigma=\int S d X$ then fixes $S_{\max }$. Explicitly,

$$
\begin{aligned}
\delta & =\{<X>/ R \operatorname{Rms}(X)\}^{2}-1 & X_{\max } & =<X>\delta /(\delta+1) \\
S_{\max } & =\sum \delta^{\delta+1} \exp (-\delta) / \Gamma(\delta+1) / X_{\max } & w & =X_{\max } / \delta
\end{aligned}
$$


It has been checked that $\langle X\rangle$ and $\rho=\operatorname{Rms}(X) /\langle X\rangle$ are not significantly correlated, thereby making it legitimate to apply independent Gaussian fluctuations to each.
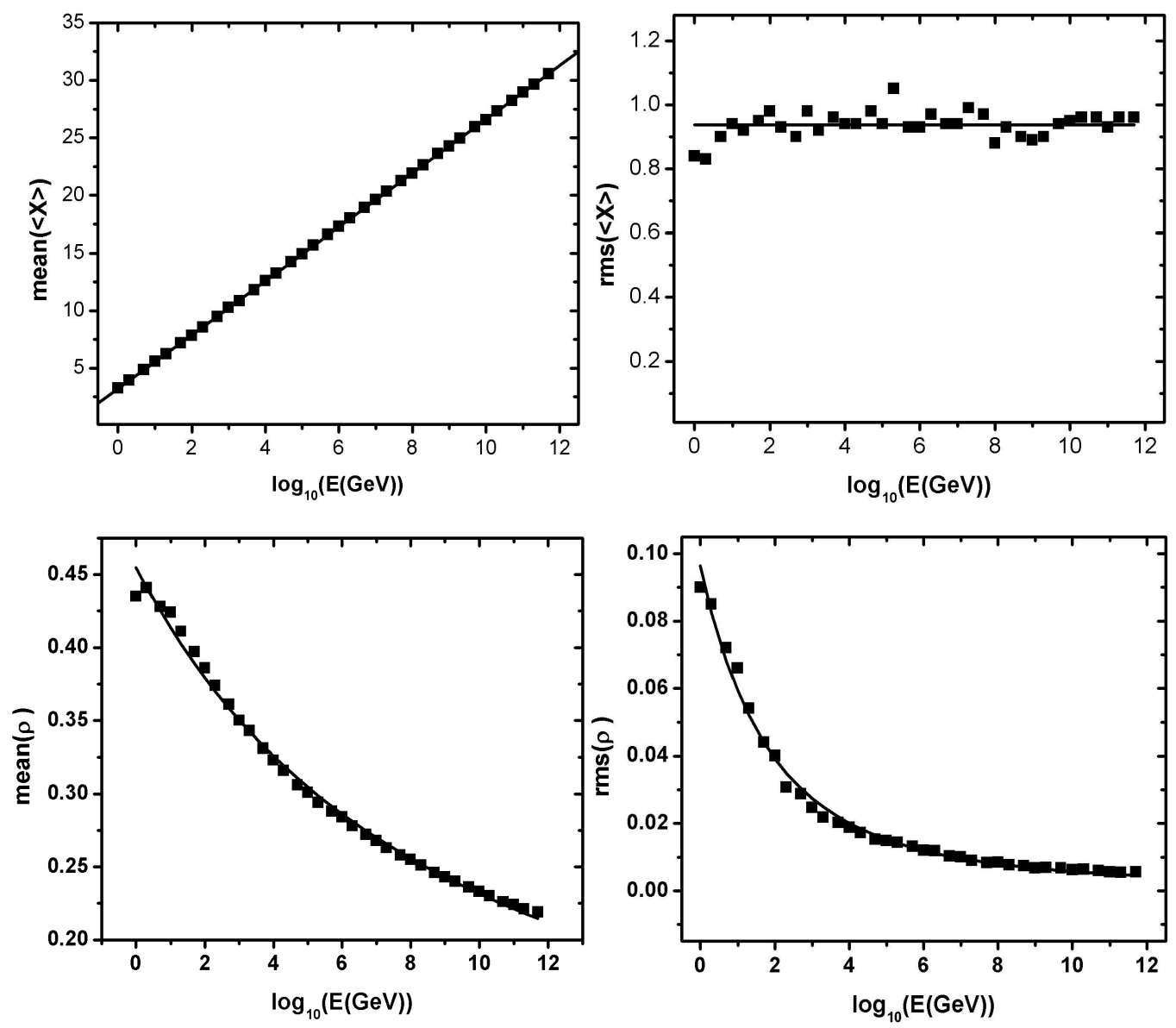

Fig. 4. Dependence on energy of the parameters defining the longitudinal shower profile. Upper panels: Mean value of $\langle X\rangle$ (left) and $r m s$ value of $\langle X\rangle$ (right); units are radiation lengths. Lower panels: Mean value of $\rho$ (left) and $\mathrm{rms}$ value of $\rho$ (right). The lines are the result of the fits described in the text.

The dependence on energy of the mean and rms values of $\langle X\rangle$ and $\rho$ evaluated by the present simulation is illustrated in Figure 4 . The parameters were calculated with full shower development up to an initial energy of $100 \mathrm{GeV}$. Above this energy, any shower particle having an energy smaller than $40 \%$ of the initial energy was replaced by a GaisserHillas profile evaluated for the proper values of the relevant parameters (after application of Gaussian fluctuations). The start of the profile was defined as $X^{*}=0$ for electrons and was chosen at random with an $\exp \left(-[7 / 9] X^{*} / X_{0}\right)$ distribution for photons. As $E_{c}=80$ 
$\mathrm{MeV}$ is the only scale of the problem, the development of the profile scales in proportion with the logarithm of the energy as soon as $E_{c}$ is negligible with respect to initial energy.

Because of shower-to-shower fluctuations, the parameters that describe the average profile (obtained as superposition of a large number of different showers) are not exactly the same as the mean values of the parameters that describe individual profiles (as displayed in Figure 4). More precisely, the mean value of the former profile, $\left\langle X^{\prime}\right\rangle$, and that of the mean values of the latter profiles, $\langle\langle X\rangle\rangle$, are equal and can be parameterized as $3.22+2.34 \log _{10} E$. But the $\rho$ parameter of the former profile, $\rho^{\prime}$, and the mean value of the $\rho$ parameters of the latter profile, $\langle\rho\rangle$, differ. They can be parameterized as

$$
\rho^{\prime}=0.102+1.91 /\left(\log _{10} E+4.246\right) \text { and }<\rho>=0.020+4.106 /\left(\log _{10} E+9.449\right)
$$

respectively. In these parameterizations, units are radiation lengths and $\mathrm{GeV}$. The difference between $\rho^{\prime}$ and $\rho$ is further illustrated in Figure 5 which compares the corresponding values of $b=1 / w=1 /\left(<X>\rho^{2}\right)$. The asymptotic value of $\sim .70$ inverse radiation lengths is reached by $b^{\prime}$ significantly earlier than by $b$.

In the case of the latter profiles, the rms values of the quantities $\langle X\rangle$ and $\rho$ define the size of the shower-to-shower fluctuations. To a very good approximation, $\operatorname{Rms}(<X>)$ is constant and equal to $0.94 \pm 0.01$ radiation lengths. On the contrary, $\operatorname{Rms}(\rho)$ is found to decrease with energy as $\operatorname{Rms}(\rho)=0.001+16.20 /\left(\log _{10} E+5.6\right)^{3}$.

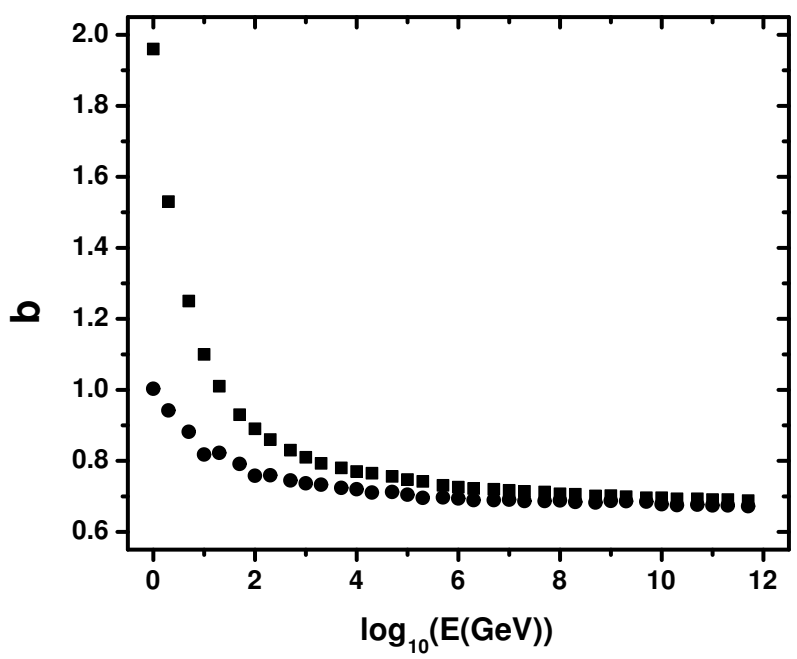

Fig. 5. Dependence of $b=1 / w=1 /\left(<X>\rho^{2}\right)$ on energy for the mean profile (full circles) and for individual profiles (full squares). Units on the ordinate are inverse radiation lengths. 


\section{LPM REDUCTION}

\section{III.1. Description of The Effect}

The unusual kinematics conditions of bremsstrahlung and pair creation are at the source of the LPM effect. In the case of bremsstrahlung by a relativistic electron, the momentum transfer, and particularly its longitudinal component, is very small. Letting $E$ be the incident electron energy and $m$ the electron mass, the longitudinal momentum transfer is (to first order in $\epsilon=m / E<<1$ and neglecting transverse momenta)

$$
\begin{aligned}
q_{L}=p_{e}-p_{e}^{\prime}-k & =\sqrt{E^{2}-m^{2}}-\sqrt{(E-k)^{2}-m^{2}}-k \\
& =E\left(1-1 / 2 \epsilon^{2}\right)-(E-k)\left\{1-1 / 2 \epsilon^{2} E^{2} /(E-k)^{2}\right\}-k \\
& =1 / 2 \epsilon^{2}\left\{-E+E^{2} /(E-k)\right\} \\
& =1 / 2 \epsilon^{2} E k /(E-k) \\
& =m^{2} k /\{2 E(E-k)\}
\end{aligned}
$$

where $p_{e}$ and $p_{e}^{\prime}$ are the electron longitudinal momenta before and after radiation took place and $k$ is the longitudinal momentum of the radiated photon.

The Heisenberg uncertainty principle implies that the formation of the final state occurs over a distance $l_{f 0}=\hbar / q_{L}=2 \hbar E(E-k) /\left(m^{2} k\right)$, called the formation length, that may be very large. As an example, a $10^{18} \mathrm{eV}$ electron radiating a $10^{15} \mathrm{eV}$ photon gives $q_{L}=10^{-10} \mathrm{eV}$ and $l_{f 0}=2 \mathrm{~km}$. Over the formation length, the system cannot be significantly disturbed for the final state to materialize. Any significant perturbation will strongly reduce the bremsstrahlung cross-section. In particular, multiple Coulomb scattering will produce such a perturbation as soon as the multiple scattering angle [3], integrated over the formation length, exceeds the characteristic bremsstrahlung emission angle $\epsilon$. This occurs over a distance $l_{m s}$ that is easily calculated. To a good approximation, the reduction factor $S$ is simply

$$
S=l_{f} / l_{f 0}=\sqrt{k E_{L P M} /(E(E-k)} \text { where }
$$

$E_{L P M}=m^{4} X_{0} /\left(2 \hbar E^{2}\right) \approx 3.85 \mathrm{TeV} / \mathrm{cm} X_{0}\left(2.2 \mathrm{TeV}\right.$ for lead and $1.1710^{17} \mathrm{eV}$ for air at sea level). To this approximation, $S$ is a universal function of the scaling variables $k / E_{L P M}$ and $E / E_{L P M}$.

While the standard bremsstrahlung cross-section is of the form $d N / d k \sim 1 / k$ the LPM reduced cross-section is instead $\sim 1 / \sqrt{k}$.

A similar effect takes place in the case of pair creation.

\section{III.2. Migdal Evaluation and Experimental Evidence}

Migdal [2] has performed a more serious evaluation of the suppression factors and his results are displayed in Figure 1 in the case of lead. When the electron energy reaches 100 $\mathrm{GeV}$ or so, bremsstrahlung starts to be significantly reduced, in particular the radiation of lower energy photons. The same happens in the case of pair creation when the photon energy reaches $10 \mathrm{TeV}$ or so, symmetric pairs being preferentially suppressed. Integrating the curves displayed in Figure 1 gives the global reduction factor. Its dependence on energy is shown in Figure 6 for both electrons (bremsstrahlung) and photons (pair creation). 


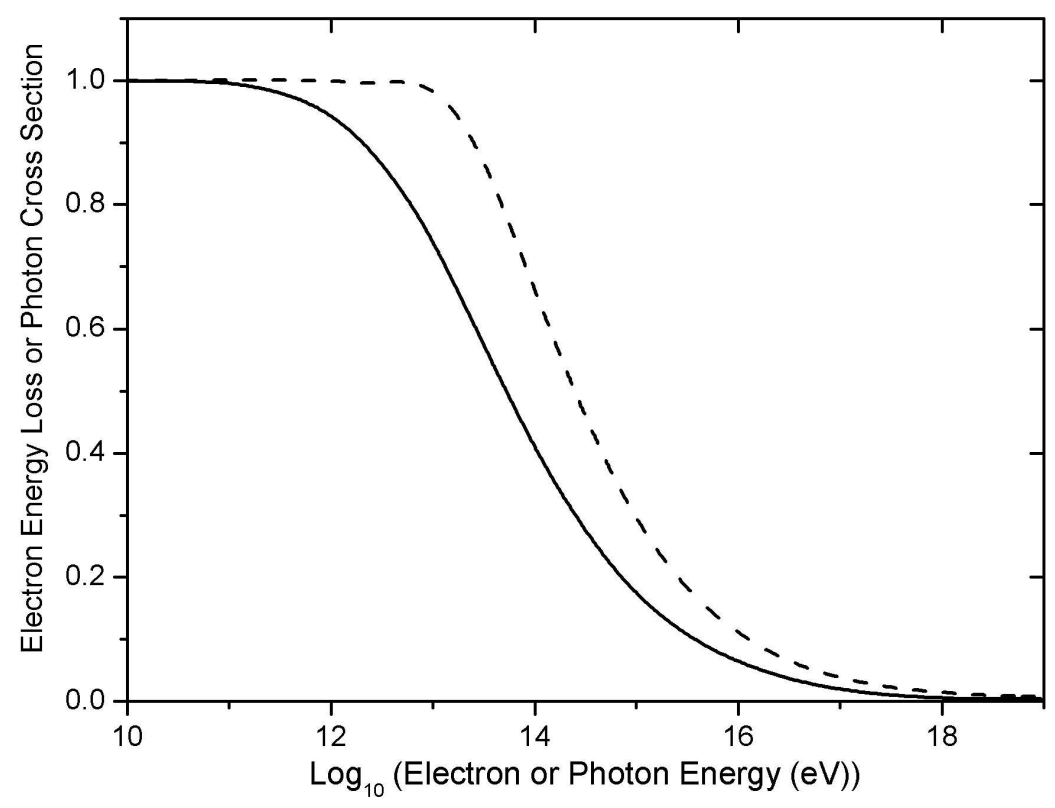

Fig. 6. Energy dependence of the LPM reduction factors in $\mathrm{Pb}$ for bremsstrahlung (full line) and pair creation (dashed line).

The LPM effect has been studied at SLAC using electrons of 8 to $25 \mathrm{GeV}$ incident on thin targets ( 0.001 to $\left.0.06 X_{0}\right)$ made of various materials (from carbon to gold) [5]. The beam was pulsed, with, on average, one electron per pulse, and bent in a magnetic dipole by $39 \mathrm{mrad}$ after having crossed the target. Both the radiated photon and the outgoing electron were detected in high resolution arrays (BGO for the photon, with a $4 \%$ energy resolution, and lead glass for the electron). Beam lines were evacuated. High quality data were collected and the $25 \mathrm{GeV}$ gold data $\left(0.06\right.$ and $0.07 X_{0}$ ) show a strong LPM reduction (up to a factor 3 for photon energies of $5 \mathrm{MeV}$ ) well described by the Migdal model. Well understood edge effects cause differences between thin and thick target data. These data give confidence in the Migdal calculation and justify the approximations made.

\section{EVALUATION OF THE EFFECT ON EXTENSIVE AIR SHOWERS}

In the case of extensive air showers, the situation is not as simple as in the case of solid targets: the density of atmosphere, and therefore the value of $E_{L P M}$ depend on altitude. In the present context it is sufficient to model the atmospheric pressure in the form of an exponential decreasing over a characteristic length of $8.7 \mathrm{~km}$. The value [6] taken by $E_{L P M}$ is $1.1710^{17} \mathrm{eV}\left(A_{0} / A\right)$, where $A$ is the thickness of air above the altitude under consideration and $A_{0}$ its value at sea level, $1030 \mathrm{~g} / \mathrm{cm}^{2} . E_{L P M}$ is therefore $3.4 \mathrm{EeV}$ for 36 $\mathrm{g} / \mathrm{cm}^{2}\left(1 X_{0}\right)$, and $1.3 \mathrm{EeV}$ for $\sim 90 \mathrm{~g} / \mathrm{cm}^{2}$ (one hadronic interaction length). Temperature effects may slightly affect these values but are ignored here. The first hadronic interaction produces several hundred pions with neutral pions decaying exponentially into a photon pair over a decay length of $\sim 200 \mathrm{~m} / \mathrm{EeV}$. The highest energy neutral pions can reach 20 
or so EeV but they represent a small fraction, a few percent at most, of the secondary pions. This dilution of the incident energy among many pions considerably reduces the impact of the LPM effect.

The changes induced by the LPM effect on the integrated (Figure 6) and differential (Figure 1) bremsstrahlung and pair creation cross-sections have been implemented in the simulation code. Calling $F_{e}$ and $F_{\gamma}$ the reduction factors displayed in Figure 6, calculated for lead with $E_{L P M}=2.2 \mathrm{TeV}$, their values for air at altitude $z$ $(\mathrm{km})$ are $F\left\{E \times E_{L P M}(\right.$ lead $) / E_{L P M}($ air $\left.)\right\}$. As $E_{L P M}($ air $)=1.1710^{17} \mathrm{eV}\left(A_{0} / A\right)$ and $A=A_{0} \exp (-z / 8.7), E_{L P M}($ air $)=1.1710^{17} \mathrm{eV} \exp (z / 8.7)$ : the reduction factors are $F\left\{E \times(2.2 / 1.17) \times 10^{-5} \times \exp (-z / 8.7)\right\}$. At the $n^{t h} 0.01 X_{0}$ step, using $A_{0}=1030 \mathrm{~g} / \mathrm{cm}^{2}$ and $X_{0}=36 \mathrm{~g} / \mathrm{cm}^{2}$, one reaches an altitude $z$ such that

$1030 \exp (-z / 8.7)=n \cos \theta 36 / 100$ where $\theta$ is the shower zenith angle. Then

$F\left\{E \times(2.2 / 1.17) \times 10^{-5} \times \exp (-z / 8.7)\right\}=F\left\{6.6 E n \cos \theta 10^{-9}\right\}$.

The LPM reduction in the upper atmosphere is therefore of the same order of magnitude as in lead at an energy a million times lower. The reduction occurs on bremsstrahlung at lower energy -typically one order of magnitude lower- than in pair creation. Results are presented in Figure 7 as a function of energy for incident electrons and for three angles of incidence: vertical, $30^{\circ}$ and $60^{\circ}$. The main effect on the differential bremsstrahlung cross-section is to suppress preferentially the radiation of low energy photons, namely to favour bremsstrahlung of high energy photons. This increases the efficiency of the cascade mechanism in the development of the shower, which tends to make it shorter and counteracts somewhat the effect of the reduction of the total cross-section which tends to make it longer. Indeed, $\langle\langle X\rangle\rangle$ is even found to decrease slightly with respect to the no LPM case before taking off significantly around $10^{21} \mathrm{eV}$ but this small decrease is not significant within the accuracy of the model calculation. The increase in $\langle\rho\rangle$, larger than that in $\langle<X\rangle>$, starts being significant above $10^{20} \mathrm{eV}$. Large zenith angle showers develop longer in the low density upper atmosphere and are therefore less affected by the LPM suppression.

The average effect on extensive air showers is therefore negligible in practical cases. However, shower-to-shower fluctuations are found to be strongly influenced by the LPM effect. They are amplified by large factors reaching, at $5.10^{20} \mathrm{eV}, 6.4,5.0$ and 2.7 for vertical, $30^{\circ}$ and $60^{\circ}$ showers respectively. The LPM effect may therefore need to be taken into account when shower-to-shower fluctuations are of particular relevance.

In practical cases, results will depend upon the altitude of the observatory as showers ending into ground are only partially measured. In the present simulation, this effect has been ignored: the altitude of the observatory was supposed to be low enough to allow the shower to fully develop in atmosphere.

The results displayed in Figure 7 apply to showers induced by electrons or photons. They would be of direct relevance to $\gamma$-ray astronomy if energies in excess of $\sim 10 \mathrm{EeV}$ could be reached, which is far from being the case.

Extensive air showers can reach such energies but the impact of the LPM effect on their development is considerably reduced by two factors. One, mentioned earlier, is the high multiplicity of the first hadronic interaction, which dilutes considerably the available energy among the secondary mesons produced. The second results from the fluctuations 

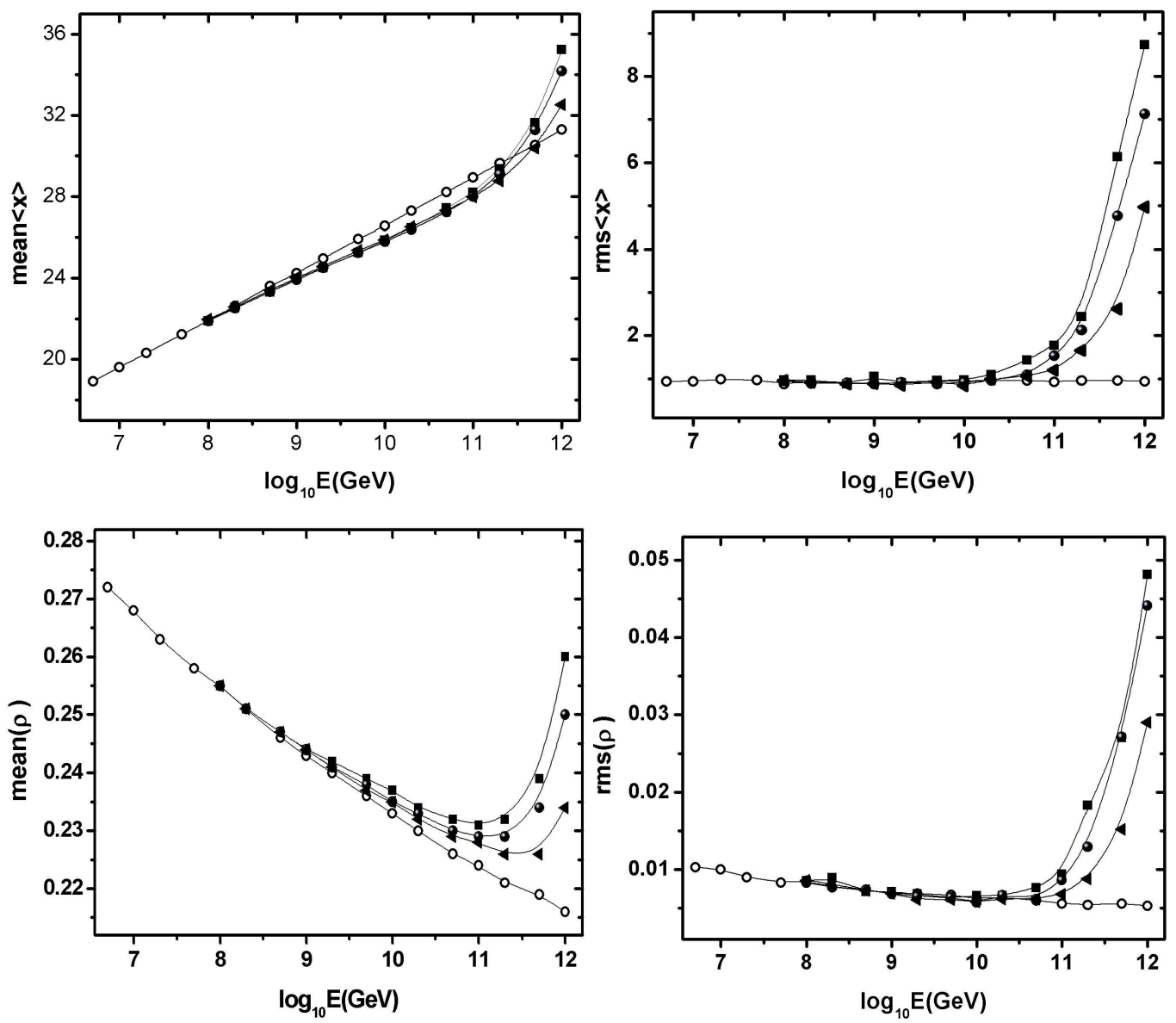

Fig. 7. Dependence on energy of the parameters calculated (LPM included) for incident electrons and for three angles of incidence: vertical (full circles), $30^{\circ}$ (full squares) and $60^{\circ}$ (full triangles). Mean values are shown in the left panels and rms values in the right panels; $\langle\langle X\rangle\rangle$ is shown in the upper panels and $\rho$ in the lower panels. The results obtained when ignoring the LPM effect are shown as open circles.

associated with the large decay length of neutral pions. The distribution of their decay vertices being exponential, the resulting shower-to-shower fluctuations have an rms value equal to the characteristic decay length, much larger than that induced by the LPM effect. In the typical case of a vertical shower produced by a first hadronic interaction at an altitude of $20 \mathrm{~km}$, one hadronic interaction length, $90 \mathrm{gcm}^{-2}$, corresponds to $5.5 \mathrm{~km}$, which in turn is the characteristic decay length of a neutral pion of $\sim 30 \mathrm{EeV}$. At such energy, the LPM effect is just starting to take off while the fluctuations associated with the neutral pion decay are already at a scale commensurate with the difference expected between showers induced by protons and respectively iron nuclei. 
In conclusion, the incidence of the LPM effect on $\gamma$-ray astronomy and on the physics of extensive air showers is very small and can be neglected in most practical cases. The parameterization of the longitudinal profile of electromagnetic showers presented here should prove useful when dealing with problems such as the LPM effect presented here as an illustration.

\section{ACKNOWLEDGEMENTS}

The present work was inspired by our association with the Pierre Auger Observatory and we thank our colleagues in this collaboration, particularly Professors P. Billoir and M. Urban, for their constant and friendly interest in our work. We acknowledge support from the World Laboratory, the Rencontres du Vietnam and Odon Vallet Fellowships, the French CNRS, the Vietnam Atomic Energy Commission and the Vietnam Ministry of Sciences and Technology.

\section{REFERENCES}

[1] K. Greisen, Phys. Rev. Lett. 16 (1966) 748;

Z.T. Zatsepin and V. A. Kuzmin, Zh. Eksp. Teor. Fiz. Pis'ma Red. 4 (1966) 144.

[2] L. D. Landau, and I. J. Pomeranchuk, Dokl. Akad. Nauk. SSSR 92 (1953) 535; Dokl. Akad. Nauk. SSSR 92 (1953) 735. These papers are available in English in The Collected Papers of L. D. Landau, Pergamon Press, 1965; A. B. Migdal, Phys. Rev. 103 (1956) 1811.

[3] Particle Data Group, S. Eidelman et al., Physics Letters B592 (2004) 1;

P.N. Diep, VATLY Internal Note 21, December 2008 and references therein.

[4] P. Sokolsky, Am. Inst. Phys. Conf. Proc. 433 (1998) 65.

[5] P. L. Anthony et al., Phys. Rev. Lett. 75 (1995) 1949.

[6] S. R. Klein, LBNL 41167, December 1997, arXiv:astro-ph/9712198v3 11 Dec 2000 and references therein.

Received 12 September 2009. 